${ }^{1}$ Unidad de Medicina Materno Fetal, División de Obstetricia y Ginecología. ${ }^{2} U$ nidad de Medicina Basada en la Evidencia. Escuela de Medicina, Pontificia Universidad Católica de Chile. Santiago, Chile.

Recibido el 11 de mayo de 2016, aceptado el 8 de septiembre de 2016.

Correspondencia a: Dr. Enrique Donoso Lira $85,5^{\circ}$ piso. Santiago, Chile. Teléfono: 223543034 edonoso@med.puc.cl

\section{Aumento de la mortalidad infantil en niños con síndrome de Down: Chile 1997-2013}

\author{
ENRIQUE DONOSO $^{1}$, CLAUDIO VERA ${ }^{1,2}$
}

\section{Rising infant mortality in down syndrome in Chile from 1997 to 2013}

Background: Down syndrome (DS) is associated with higher child mortality especially due to cardiac malformations. Aim: To describe the trend in Chilean infant mortality in DS in the period 1997-2013 as compared to the general population without DS. Material and Methods: Raw data on infant deaths were extracted from the yearbooks of vital statistics of the National Institute of Statistics. The mortality risk associated to DS, relative to population without DS was estimated. Results: There were 456 deaths in infants with DS during the study period (59 early neonatal deaths, 70 late neonatal deaths and 327 post-neonatal deaths). The trend in infant mortality rate in DS was ascending $(r: 0.53, p=0.03)$, with an average annual percentage change of $4.6 \%(95 \%$ confidence interval (CI) $0.4-9.0 \%$; $p<0.01$ ). Compared to the population without DS, the risk of early neonatal death was lower in DS (Odds ratio (OR) $0.14,95 \%$ CI 0.11-0.19; $p<0.01)$ whereas the risk of post-neonatal death was higher (OR 4.74, 95\% CI 3.85-5.85; $p<0.01)$. Conclusions: Infant mortality in Down syndrome has an increasing trend. We postulate that these children are not accessing timely cardiac surgery, the main therapeutic tool to reduce the death risk in the first year of life.

(Rev Med Chile 2016; 144: 1432-1439)

Key words: Chile; Down Syndrome; Heart Defects, Congenital; Infant Mortality.
$\mathrm{E}$ 1 síndrome de Down (SD) es la anomalía congénita no letal ${ }^{1}$ y de discapacidad mental más frecuente ${ }^{2}$. El SD está asociado a defectos estructurales mayores ${ }^{3}$ y otras comorbilidades que condicionan una menor sobrevida en el primer año de vida ${ }^{4}$ y en las otras etapas del ciclo vital ${ }^{5-7}$. El defecto estructural asociado más frecuente son las cardiopatías congénitas (40-45\%), especialmente los defectos del tabique aurículo-ventricular ${ }^{3,8,9}$.

En Chile se observa un cambio demográfico importante, caracterizado por un aumento de los nacimientos en mujeres 40 o más años ${ }^{10} \mathrm{y}$ un envejecimiento de las mujeres chilenas en edad fértil ${ }^{11}$, que las expone a un mayor riesgo de malformaciones congénitas y de hijos con síndrome de Down. Es así, que nuestro país tiene la tasa más alta de SD en Latinoamérica ${ }^{12}$ y con tendencia al ascenso $^{13,14}$.

En Chile, la interrupción del embarazo es ilegal en cualquier circunstancia ${ }^{15}$, por lo que el conocimiento de la prevalencia del SD y tasas de mortalidad, reflejarían la historia natural de esta condición genética y permitiría formular políticas sanitarias que favorezcan su inclusión a la sociedad y el tratamiento oportuno de las comorbilidades.

Chile carece de un registro nacional de malformaciones congénitas y anomalías cromosó- 
micas, con excepción de informes nacionales de hospitales asociados al Estudio Colaborativo Latino Americano de Malformaciones Congénitas $(\text { ECLAMC })^{16}$. Es así, que la prevalencia de SD registrada en el período 2001-2010 en 13 hospitales chilenos fue de 25/10.000 nacimientos sobre un total de 282.568 nacimientos (nacidos muertos y vivos $)^{17}$. Extrapolando esa tasa a los nacimientos ocurridos en Chile en 2013 (2.090 muertes fetales y 242.862 nacidos vivos) $)^{18}$, se esperaría 612 casos de SD para ese año. Los niños con SD son de alto riesgo de morbilidad y mortalidad comparado con la población infantil general, generando una mayor frecuencia de controles pediátricos y hospitalizaciones en unidades de cuidados intensivos $^{4,19,20,21}$. La cirugía cardiaca especialmente, y la atención oportuna y eficaz del tratamiento de las comorbilidades ha permitido a los niños con SD una sobrevida significativa al año de vida ${ }^{22,23}$, alcanzando en algunas series una sobrevida post-cirugía cardiaca superior a $90 \% \%^{9,21,24}$.

El objetivo de este estudio es analizar en Chile las muertes durante el primer año de vida de niños con SD, como también sus componentes neonatales precoz, neonatal tardío y pos neonatal, comparadas con la población general no SD, en el período 1997-2013.

\section{Material y Métodos}

Análisis poblacional, retrospectivo, de la mortalidad infantil y de sus componentes etarios en niños con SD, comparándola con la de población general sin SD, en el período 1997-2013. El período de estudio fue seleccionado por la incorporación, en 1997, de la Décima Revisión de la Clasificación Internacional de Enfermedades y Problemas Relacionados con la Salud (CIE 10) ${ }^{25}$. El SD está identificado en CIE 10 bajo el código Q90. Los datos crudos fueron extraídos desde los Anuarios de Demografía y Estadísticas Vitales publicados por el Instituto Nacional de Estadísticas (INE) correspondientes al período de estudio ${ }^{26}$. En ambos grupos se analizó la edad al fallecimiento considerando las muertes en los primeros 7 días de vida (muerte neonatal precoz), entre los 7 y 27 días de vida (muerte neonatal tardía), y entre los 28 días y 11 meses (mortalidad pos neonatal). La tasa de mortalidad infantil y de sus componentes etarios en niños con SD se expresó por 100.000 nacidos vivos (nv) corregidos en los años correspondientes al estudio y en la población general de niños sin SD por $1.000 \mathrm{nv}$, excluidos los casos de SD. La estimación del riesgo de morir asociado a SD, relativo al grupo sin esta condición, se efectuó mediante el cálculo del Odds Ratio (OR) con intervalo de confianza de 95\% (IC 95\%). El estudio de tendencia de la mortalidad infantil y sus componentes etarios se efectuó según análisis de correlación de Pearson (2 colas). Se estimó el cambio porcentual anual de las tasas mediante un modelo de regresión log-linear para el logaritmo natural de la tasa (y) en el año (x); el modelo estima el cambio porcentual anual como $e^{\beta_{1}}-1$ y el cambio porcentual anual promedio (CPAP) del período considerando los segmentos de diferente tendencia en la serie completa, se reporta el IC $95 \%$ y el valor $\mathrm{p}$ para cambios de CPAP significativamente distintos de 0 con alfa $0,05^{27}$.

\section{Resultados}

En el período 1997-2013 hubo 4.187.567 recién nacidos vivos corregidos sin SD, 35.111 muertes infantiles $(8,3 / 1.000 \mathrm{nv}), 17.905$ muertes neonatales precoces $(4,3 / 1.000 \mathrm{nv}), 4.977$ muertes neonatales tardías $(1,2 / 1.000 \mathrm{nv})$, y 12.229 muertes pos neonatales $(2,9 / 1.000 \mathrm{nv})$. El 51,0\% de las muertes ocurren el período neonatal precoz, $14,2 \%$ en el período neonatal tardío y $34,8 \%$ en el período pos neonatal. La tendencia de la tasa de mortalidad infantil en niños sin SD fue significativamente descendente ( $\mathrm{r}:-0,9067 ; \mathrm{p}<0,001$ ), la tasa de mortalidad neonatal precoz sin cambios significativos ( $\mathrm{r}:-0,4131 ; \mathrm{p}=0,12$ ), la tasa de mortalidad neonatal tardía descendió significativamente ( $\mathrm{r}$ : $-0,7824, p=0,001)$ y la tasa de mortalidad pos neonatal descendió significativamente ( $\mathrm{r}$ : $-0,9517$; $\mathrm{p}<0,001$ ) (Figura 1). La mortalidad infantil de la población general de niños sin SD presentó un cambio porcentual anual promedio descendente significativo de $-2,5 \%(-3,4 \mathrm{a}-1,6 \% ; \mathrm{p}<0,001)$.

En niños con SD hubo 456 muertes infantiles $(10,9 / 100.000 \mathrm{nv}), 59$ muertes neonatal precoces $(1,4 / 100.000 \mathrm{nv}), 70$ muertes neonatales tardías $(1,7 / 100.000 \mathrm{nv})$, y 327 muertes pos neonatales $(7,8 / 100.000 \mathrm{nv})$. El 12,9\% de las muertes ocurren el período neonatal precoz, $15,4 \%$ en el período neonatal tardío y $71,7 \%$ en el período pos neonatal. La tendencia de la tasa de mortalidad infantil 


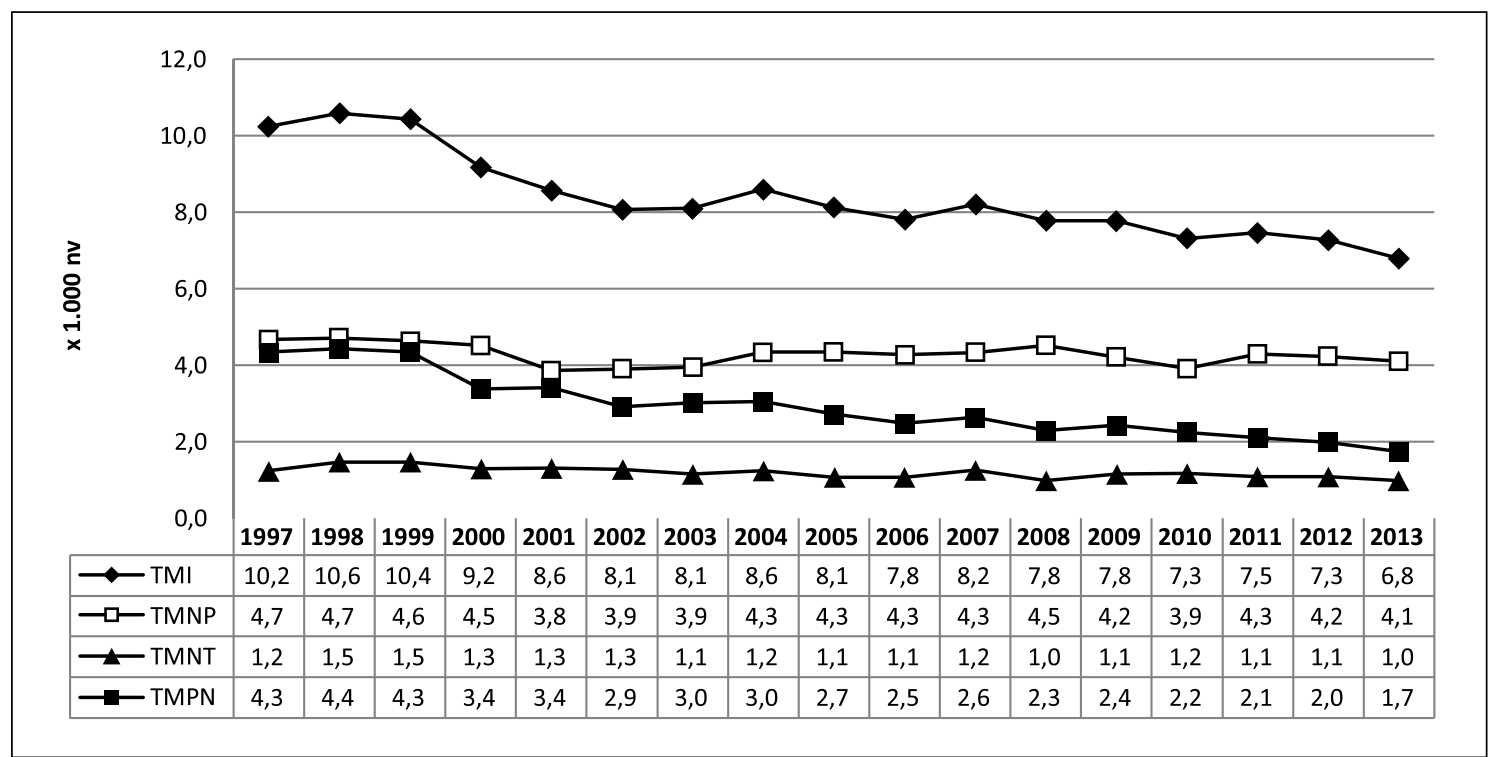

Figura 1. Tendencia de la tasa de la mortalidad infantil (TMI), mortalidad neonatal precoz (TMNP), mortalidad neonatal tardía (TMNT) y mortalidad posneonatal (TMPN) en población general excluida las por síndrome de Down.

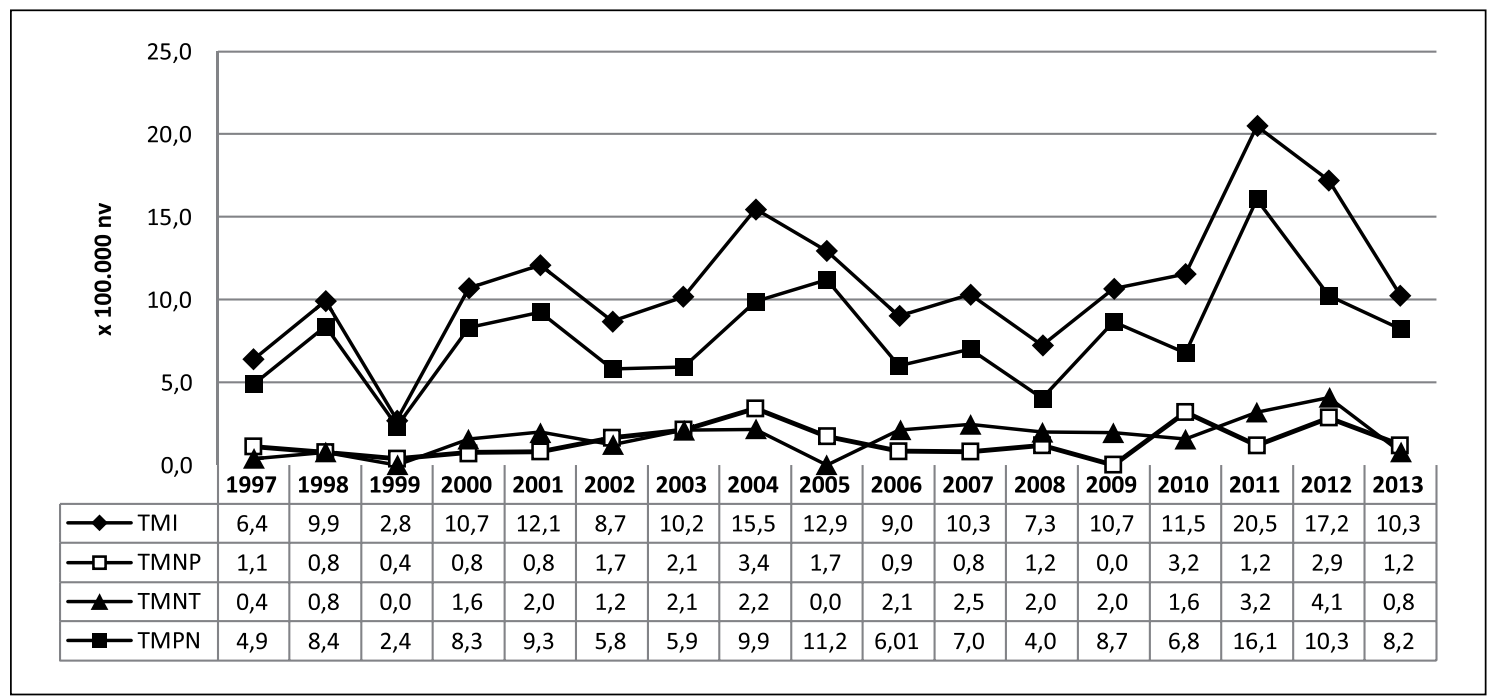

Figura 2. Tendencia de la tasa de la mortalidad infantil (TMI), mortalidad neonatal precoz (TMNP), mortalidad neonatal tardía (TMNT) y mortalidad posneonatal (TMPN) en población portadora de síndrome de Down.

en niños con SD fue significativamente ascendente ( $\mathrm{r}: 0,5327 ; \mathrm{p}=0,028)$, la tasa de mortalidad neonatal precoz sin cambios significativos ( $\mathrm{r}$ : 0,2892; $\mathrm{p}=0,26)$, la tasa de mortalidad neonatal tardía ascendió significativamente ( $\mathrm{r}: 0,5592 ; \mathrm{p}=0,021)$, y la tasa de mortalidad pos neonatal no presentó cambios significativos (r: 0,4118; p = 0,10) (Fi- gura 2). La mortalidad infantil de niños con SD presentó un cambio porcentual anual promedio ascendente significativo de $4,6 \%(0,4$ a $9,0 \%$; $p<0,001)$. No hubo diferencias significativas en la proporción de muertes de niños con SD según sexo en ninguno de los 3 períodos del primer año de vida (Tabla 1). 
Tabla 1. Muerte neonatal precoz, neonatal tardía, posneonatal e infantil asociadas a síndrome de Down según sexo del recién nacido. Chile 1997-2013

\begin{tabular}{|lcccc|}
\hline Sexo & \multicolumn{2}{c}{ Componentes de la mortalidad infantil } \\
Neonatal precoz & $\begin{array}{c}\text { Neonatal tardía } \\
\mathbf{n ~ ( \% )}\end{array}$ & $\begin{array}{c}\text { Posneonatal } \\
\mathbf{n}(\%)\end{array}$ & $\begin{array}{c}\text { I\%) } \\
\text { Infantil } \\
\mathbf{n}(\%)\end{array}$ \\
\hline Masculino & $36(15,52)$ & $34(14,66)$ & $162(69,83)$ & $232(100)$ \\
\hline Femenino & $23(10,27)$ & $36(16,07)$ & $165(73,66)$ & $224(100)$ \\
\hline Total & $59(12,94)$ & $70(15,35)$ & $327(71,71)$ & $456(100)$ \\
OR; IC 95\% & 1,$61 ; 0,91-2,87$ & 0,$94 ; 0,56-1,57$ & 0,$97 ; 0,73-1,29$ & 1 \\
\hline
\end{tabular}

Tabla 2. Muerte neonatal precoz, neonatal tardía, posneonatal e infantil asociadas a SD vs población general no asociada a síndrome de Down. Chile 1997-2013

\begin{tabular}{|c|c|c|c|c|}
\hline \multirow[b]{2}{*}{ Condición } & \multicolumn{3}{|c|}{ Componentes de la mortalidad infantil } & \multirow[b]{2}{*}{$\begin{array}{c}\text { Infantil } \\
\text { n (\%) }\end{array}$} \\
\hline & $\begin{array}{c}\text { Neonatal precoz } \\
\text { n (\%) }\end{array}$ & $\begin{array}{c}\text { Neonatal tardía } \\
\text { n (\%) }\end{array}$ & $\begin{array}{c}\text { Posneonatal } \\
\text { n (\%) }\end{array}$ & \\
\hline Down & $59(12,94)$ & $70(15,35)$ & $327(71,71)$ & $456(100)$ \\
\hline No Down & $17.905(51,00)$ & $4.977(14,18)$ & $12.229(34,83)$ & $35.111(100)$ \\
\hline Total & $17.964(50,51)$ & $5.047(14,19)$ & $12.556(35,30)$ & $35.567(100)$ \\
\hline OR; IC 95\% & $0,14(0,11-0,19)$ & $1,10(0,84-1,43)$ & $4,74(3,85-5,85)$ & 1 \\
\hline
\end{tabular}

Durante el primer año de vida, comparado con la población de niños sin SD; el riesgo de muerte neonatal precoz fue menor en niños con SD (OR $0,14$; IC 95\% 0,11 a 0,19; $p=0,001)$; no se observó diferencia significativa en el riesgo de muerte neonatal tardía (OR 1,10; IC 95\% 0,84 a 1,43), mientras que el riesgo de muerte pos neonatal fue mayor en niños con SD (OR 4,74; IC 95\% 3,85 a $5,85 ; \mathrm{p}=0,001)($ Tabla 2$)$.

\section{Discusión}

La prevalencia de nacidos vivos con SD es muy variable entre países y depende de la proporción de embarazos en edad materna avanzada (35 o más años) ${ }^{28,29}$, del uso de técnicas de diagnóstico antenatal de malformaciones y anomalías cromosómicas ${ }^{30,31}$ y del aborto eugenésico legal ${ }^{12-34}$.

Nuestra investigación permitió determinar la mortalidad infantil y de sus componentes en niños con SD en un período de 17 años en Chile, información no conocida anteriormente. Observamos una tendencia ascendente significativa de la mortalidad infantil en niños con SD que contrasta con la tendencia descendente significativa de la mortalidad infantil de población general sin SD. Se pudo determinar que no hay diferencias significativas en la proporción de muertes en niños con $\mathrm{SD}$ en el primer año de vida según sexo de los fallecidos, lo que permite el análisis global de los resultados. Los análisis están limitados a la información disponible no siendo posible ajustar por otros potenciales factores confundentes.

Se destaca que la proporción de muertes en los diferentes períodos del primer año de vida es distinto entre los grupos, y caracterizada por menor riesgo de muerte en niños con SD en el período neonatal precoz (OR 0,14; IC 95\% 0,11 a 0,19$)$, mientras que en el período pos neonatal esta relación se invierte, siendo el riesgo de muerte en niños con SD significativamente mayor (OR 4,74; IC 95\% 3,85 a 5,85). Lo anterior se puede explicar porque en la población general de niños sin SD se incluyen otras condiciones de riesgo de mortalidad neonatal precoz como malformaciones severas (polimalformados, anencefalia, cardiopatías congénitas complejas, agenesia renal, etc.), la 
asfixia perinatal grave, los prematuros extremos, $y$ las trisomías 13 y 18 entre otras, las que no fueron excluidas en el actual estudio. Esto se confirma en un estudio nacional correspondiente al período 1991-1997 que mostró que 55,8\% de la muertes neonatales precoces ocurren en el primer día de vida, siendo las primeras causas, las malformaciones congénitas y las anomalías cromosómicas $(32,3 \%)$, seguidas de los trastornos relacionados con la duración corta de la gestación y el bajo peso al nacer $(23,8 \%)$, del síndrome de dificultad respiratoria $(10,1 \%)$, la asfixia al nacer $(7,9 \%)$ y las infecciones propias del período perinatal $(4,4 \%)^{35}$.

Otro estudio nacional correspondiente al año 2008 , mostró que $33,8 \%$ de las muertes infantiles se asocian a malformaciones congénitas y a anormalidades cromosómicas; $58,4 \%$ en el período neonatal precoz, $13,5 \%$ en el neonatal tardío y $28,1 \%$ en el pos neonata ${ }^{32}$. En el actual estudio, las muertes en la población general sin SD fue 50,51\%, $14,18 \%$ y $34,83 \%$ respectivamente, semejante a lo descrito para el 2008, mientras que las muertes en niños con SD la proporción de fallecimientos en esos períodos fue de $12,94 \%, 15,35 \%$ y $71,71 \%$ respectivamente, lo que refleja el bajo impacto cuantitativo de las muertes por SD en la mortalidad neonatal precoz general y en la tendencia de la mortalidad infantil general.Un estudio reciente que analiza la tendencia secular de la mortalidad infantil en Chile de las malformaciones congénitas y anomalías cromosómicas en 3 períodos (1997-2001, 2002-2006, 2007-2011), muestra un aumento significativo de la tendencia de la mortalidad infantil por SD (8,5/100.000; 11,3/100.000; $12,1 / 100.000)$, atribuible al envejecimiento de la mujer chilena en edad fértil y al aumento de los nacimientos en edad materna avanzada ${ }^{14}$. Resultado consistente con lo observado en nuestra investigación.

Respecto a la sobrevida en el primer año de vida de los nacidos vivos con SD los datos notificados por el $\mathrm{INE}^{26}$ no permiten reconstruir esa información. Un estudio colaborativo sudamericano mostró una sobrevida de 73,6\% en el primer año de vida ${ }^{36}$, mientras que en países desarrollados esta oscila entre 91 y $96 \%$ 4,22,23,37,38. Son evidentes los mejores resultados de sobrevida infantil en los niños con SD en los países desarrollados, y puede ser consecuencia del diagnóstico precoz de malformación cardiaca y de cirugía correctora oportuna y de amplia cobertura. El aumento de la tendencia de la mortalidad infantil en niños chilenos con SD puede estar reflejando que estos no están accediendo oportunamente a cirugía cardiaca, principal intervención terapéutica de sobrevida infantil.

En Chile, no hay estudios poblacionales que describan las comorbilidades asociadas a SD y morbimortalidad infantil, con excepción de escasos estudios institucionales ${ }^{20,21}$. En un estudio nacional prospectivo de 33 niños con SD efectuado en 2006, 64,5\% presentaba cardiopatía congénita y $35 \%$ requirió cirugía cardiaca, con una sobrevida total de $93,6 \%$ al año de vida ${ }^{20}$, cifra comparable con los estudios internacionales ya descritos $^{4,22,23,37,38}$. Otro estudio nacional presenta las cardiopatías congénitas más frecuentes asociadas a SD y que requirieron corrección quirúrgica (73,5\%): canal aurículo-ventricular (45/132), ductus arterioso persistente (DAP) (17/132), comunicación interventricular (CIV) más comunicación interauricular (CIA) (12/132), CIV más DAP (11/132), CIA aislada (11/132), CIV aislada (10/132), CIA+CIV+DAP (10/132), tetralogía de Fallot (10/132), CIA+DAP (2/132) y otros (4/132); la mediana de edad de los niños operados fue de 4 meses de vida, y sin muertes atribuibles a la cirugía cardiaca ${ }^{21}$. Un tercer estudio nacional presenta los resultados de la cirugía cardiaca sobre 1.344 pacientes menores de 18 años portadores de cardiopatías congénitas, 222 de los cuales se asociaban a SD, con una mortalidad global del estudio de 5,9\% y comparable con series internacionales; no describe los resultados del grupo con $\mathrm{SD}^{39}$. Los resultados de estos estudios muestran que en Chile la atención oportuna de los niños con SD tiene excelentes expectativas de sobrevida en el primer año de vida, cuando acceden a corregir sus malformaciones cardiacas ${ }^{40}$, reduciendo el riesgo de muerte de la principal causa de mortalidad en niños con SD en el primer año de vida, como también el cuidado integral de su salud ${ }^{41}$. Sorprende que $71,7 \%$ de las muertes en niños con SD se concentren entre los 28 días y 11 meses de vida, significativamente mayor que las muertes en niños sin SD en el mismo período (OR 4,74; IC $95 \% 3,85-5,85)$. No tenemos una explicación para esta situación, pero esa diferencia pudiese corresponder a cardiopatías congénitas no corregidas en el primer año de vida, además de otras morbilidades causales de muerte $e^{3,4,19,-21}$, especialmente enfermedades respiratorias. Como presentamos 
inicialmente se esperaría alrededor de 600 casos de síndrome de Down al año, de acuerdo a los datos de prevalencia, $45 \%$ de ellos ( 270 casos) serían portadores de algún tipo de cardiopatía de los cuales $\sim 70 \%$ requerirían cirugía cardiaca ( $\sim 189$ casos) dentro del primer año de vida. Desconocemos cuántos niños con síndrome de Down han sido incorporados e intervenidos por la Ley 19.966 que aprueba las Garantías Explícitas en Salud (GES) vigente desde el 31 de enero de $2007^{42}$. De acuerdo a las guías clínicas del MINSAL ${ }^{43}$, los niños afectados con canal aurículo-ventricular completo, malformación cardíaca más frecuente en $\mathrm{SD}$, deben ser resueltos quirúrgicamente dentro de los primeros 6 meses de vida dado el riesgo de desarrollo precoz de hipertensión pulmonar, insuficiencia cardiaca y muerte ${ }^{44,45}$. Los avances en cirugía cardiaca hacen que el pronóstico de sobrevida en pacientes con SD sea excelente y semejante a los cardiópatas congénitos sin $\mathrm{SD}^{9,21,24,46}$, por lo que no se debe postergar a los niños cardiópatas con SD por su condición genética ${ }^{47}$ y se les debe ofrecer cirugía oportuna tal como establece el Ministerio de Salud de Chile ${ }^{42,43}$.

\section{Conclusión}

En Chile, en el período 1997-2013, se demuestra un aumento significativo de la mortalidad infantil en niños con síndrome de Down, postulamos que los afectados con cardiopatía no están accediendo oportunamente a cirugía cardiaca, principal herramienta terapéutica para estos niños. Futuros estudios son necesarios para confirmar la hipótesis y/o evidenciar los factores a intervenir que permitan revertir la tendencia actual de la mortalidad infantil en síndrome de Down.

\section{Referencias}

1. Parker SE, Mai CT, Canfield MA, Rickard R, Wang Y, Meyer RE, et al; National Birth Defects Prevention Network. Updated National Birth Prevalence estimates for selected birth defects in the United States, 2004-2006. Birth Defects Res A Clin Mol Teratol 2010; 88 (12): 1008-16.

2. Leonard H, Wen X. The epidemiology of mental retardation: challenges and opportunities in the new millennium. Ment Retard Dev Disabil Res Rev 2002; 8 (3): 117-34.
3. Morris JK, Garne E, Wellesley D, Addor MC, Arriola L, Barisic I, et al. Major congenital anomalies in babies born with Down syndrome: a EUROCAT population-based registry study. Am J Med Genet A 2014; 164A (12): 2979-86.

4. Weijerman ME, van Furth AM, Vonk Noordegraaf A, van Wouwe JP, Broers CJ, Gemke RJ. Prevalence, neonatal characteristics, and first-year mortality of Down syndrome: a national study. J Pediatr 2008; 152 (1): 15-9.

5. Tenenbaum A, Chavkin M, Wexler ID, Korem M, Merrick J. Morbidity and hospitalizations of adults with Down syndrome. Res Dev Disabil 2012; 33 (2): 435-41.

6. Hill DA, Gridley G, Cnattingius S, Mellemkjaer L, Linet M, Adami HO, et al. Mortality and cancer incidence among individuals with Down syndrome. Arch Intern Med 2003; 163 (6): 705-11.

7. Sobey CG, Judkins CP, Sundararajan V, Phan TG, Drummond GR, Srikanth VK. Risk of Major Cardiovascular Events in People with Down Syndrome. PLoS One 2015; 10 (9): e0137093.

8. Baraona F, Gurvitz M, Landzberg MJ, Opotowsky AR. Hospitalizations and mortality in the United States for adults with Down syndrome and congenital heart disease. Am J Cardiol 2013; 111 (7): 1046-51.

9. Irving CA, Chaudhari MP. Cardiovascular abnormalities in Down's syndrome: spectrum, management and survival over 22 years. Arch Dis Child 2012; 97 (4): 326-30.

10. Donoso E, Becker J, Villarroel L. Evolución de la natalidad y del riesgo reproductivo en mujeres de 40 o más años en la década de los 90. Rev Chil Obstet Ginecol 2002; 67 (2): 139-42.

11. Donoso E, Carvajal J, Domínguez MA. [Fecundity reduction and aging in fertile women population in the period 1990-2004 in Chile]. Rev Med Chile 2009; 137 (6): 766-73. [Article in Spanish].

12. Nazer J, Cifuentes L. [Congenital malformations in Latin America in the period 1995-2008]. Rev Med Chile 2011; 139 (1): 72-8. [Article in Spanish].

13. Nazer J, Cifuentes L. Estudio epidemiológico global del síndrome de Down. Rev Chil Pediatr 2011; 82 (2): 10512.

14. Dipierri JE, Acevedo NE, Bronberg RA. [Infant mortality from congenital malformations in Chile: temporal and spatial analysis, 1997-2011].Rev Panam Salud Publica 2015; 38 (5): 380-7. [Article in Spanish].

15. Código Sanitario, República de Chile. Libro V. Del ejercicio de la medicina y profesiones afines. Artículo 119 Ley No 18.826 del 15 de septiembre de 1989.

16. Estudio Colaborativo Latino Americano de Malforma- 
ciones Congénitas (ECLAMC). Disponible en: http:// www.eclamc.org/. [Consultado el 6 de marzo de 2016].

17. Nazer J, Cifuentes L. [Prevalence of congenital malformations at birth in Chilean maternity hospitals]. Rev Med Chile 2014; 142 (9): 1150-6. [Article in Spanish].

18. Instituto Nacional de Estadísticas (NE), Servicio de Registro Civil e Identificación (SRCeI), Departamento de Estadísticas e Información en Salud (DEIS). Estadísticas Vitales. Anuario 2013. Disponible en: http://www.ine.cl/ canales/menu/publicaciones/calendario_de_publicaciones/pdf/completa_vitales_2013.pdf. [Consultado el 23 de noviembre de 2015].

19. Fitzgerald P, Leonard H, Pikora TJ, Bourke J, Hammond G. Hospital admissions in children with Down syndrome: experience of a population-based cohort followed from birth. PLoS One 2013; 8 (8): e70401.

20. Retamales N, Moreno R, González A, Cerda J, Lizama M. Morbilidad y mortalidad durante el primer año de vida en pacientes con síndrome de Down. Rev Chil Pediatr 2009; 80 (4): 323-31.

21. Lizama Calvo M, Cerda Lorca J, Monge Iriarte M, Carrillo Mayanquer I, Clavería Rodríguez C, Castillo Moya A. [Hospital morbidity and mortality in children with Down's syndrome: Experience in a university hospital in Chile].Rev Chil Pediatr 2015; 87 (2): 102-9. [Article in Spanish].

22. Kucik JE, Shin M, Siffel C, Marengo L, Correa A; Congenital Anomaly Multistate Prevalence and Survival Collaborative. Trends in survival among children with Down syndrome in 10 regions of the United States. Pediatrics 2013; 131 (1): e27-36.

23. Glasson EJ, Jacques A, Wong K, Bourke J, Leonard H. Improved Survival in Down Syndrome over the Last 60 Years and the Impact of Perinatal Factors in Recent Decades.J Pediatr 2016; 169: 214-20.e1.

24. Frid C, Björkhem G, Jonzon A, Sunnegårdh J, Annerén G, Lundell B. Long-term survival in children with atrioventricular septal defect and common atrioventricular valvar orifice in Sweden. Cardiol Young 2004; 14 (1): 24-31.

25. Organización Mundial de la Salud (OMS), Organización Panamericana de la Salud (OPS). Clasificación Estadística Internacional de Enfermedades y Problemas Relacionados con la Salud. Decima Revisión. 1995. Publicacion Científica No 554. Disponible en: http://apps.who.int/ classifications/apps/icd/icd10online/. [Consultado el 5 de abril de 2014].

26. Instituto Nacional de Estadísticas (INE), Servicio de Registro Civil e Identificación (SRCeI), Departamento de Estadísticas e Información en Salud (DEIS). Anuarios de Demografía de 1997 a 1999. Anuarios de Estadísticas
Vitales de 2000 a 2013. Disponibles en: http://www.ine. cl/canales/chile_estadistico/familias/demograficas_vitales.php. [Consultado el 23 de noviembre de 2015].

27. Kim HJ, Fay MP, Feuer EJ, Midthune DN. Permutation tests for join point regression with applications to cancer rates. Stat Med 2000; 19 (3): 335-51. Erratum in: Stat Med 2001; 20 (4): 655.

28. Nazer J, Cifuentes L, Águila A, Ureta P, Bello MP, Correa F, Melibosky F. [The association between maternal age and congenital malformations]. Rev Med Chile 2007; 135 (11): 1463-9. [Article in Spanish].

29. Wu J, Morris JK. Trends in maternal age distribution and the live birth prevalence of Down's syndrome in England and Wales: 1938-2010.Eur J Hum Genet 2013; 21 (9): 943-7. Erratum in: Eur J Hum Genet 2013; 21 (9): 1033-4.

30. Mansfield C, Hopfer S, Marteau TM. Termination rates after prenatal diagnosis of Down syndrome, spina bifida, anencephaly, and Turner and Klinefelter syndromes: a systematic literature review. European Concerted Action: DADA (Decision-making After the Diagnosis of a fetal Abnormality). Prenat Diagn 1999; 19 (9): 808-12.

31. Natoli JL, Ackerman DL, McDermott S, Edwards JG. Prenatal diagnosis of Down syndrome: a systematic review of termination rates (1995-2011). Prenat Diagn 2012; 32 (2): 142-53.

32. Donoso E, Carvajal JA. [Eugenic abortion could explain the lower infant mortality in Cuba compared to that in Chile]. Rev Med Chile 2012; 140 (8): 999-1005. [Article in Spanish].

33. Pazol K, Creanga AA, Jamieson DJ; Centers for Disease Control and Prevention (CDC). Abortion Surveillance-United States, 2012. MMWR Surveill Summ 2015; 64 (10): 1-40.

34. Méndez-Rosado LA, Hechavarría-Estenoz D, de la Torre ME, Pimentel-Benítez H, Hernández-Gil J, Pérez B, et al. Current status of prenatal diagnosis in Cuba: causes of low prevalence of Down syndrome. Prenat Diagn 2014; 34 (11): 1049-54.

35. Donoso E, Villarroel L. Muerte neonatal precoz. Rev Chil Obstet Ginecol 1999; 64 (4): 286-91.

36. Castilla EE, Rittler M, Dutra MG, López-Camelo JS, Campaña H, Paz JE, Orioli IM. Survival of children with Down syndrome in South America. ECLAMC-Downsurv Group. Latin American Collaborative Study of Congenital Malformations. Am J Med Genet 1998; 79 (2): 108-11.

37. Leonard S, Bower C, Petterson B, Leonard H. Survival of infants born with Down's syndrome: 1980-96. Paediatr Perinat Epidemiol 2000; 14 (2): 163-71. 
38. Rankin J, Tennant PW, Bythell M, Pearce MS. Predictors of survival in children born with Down syndrome: a registry-based study. Pediatrics 2012; 129 (6): e1373-81.

39. Clavería C, Cerda J, Becker P, Schiele C, Barreno B, Urcelay G, et al. Mortalidad operatoria y estratificación de riesgo en pacientes pediátricos operados de cardiopatía congénita: experiencia de 10 años. Rev Chil Cardiol 2014; 33 (1): 11-9.

40. Miodrag N, Silverberg SE, Urbano RC, Hodapp RM. Deaths among children, adolescents, and young adults with Down syndrome. J Appl Res Intellect Disabil 2013; 26 (3): 207-14.

41. Lizama CM, Retamales MN, Mellado SC. [Recommendations for health care of people with Down syndrome from 0 to 18 years of age]. Rev Med Chile 2013; 141 (1): 80-9. [Article in Spanish].

42. Superintendencia de Salud. Gobierno de Chile. Decreto Supremo No 44 de 2007 del Ministerio de Salud: Aprueba Garantías Explícitas en Salud del Régimen General de Garantías en Salud. Publicado en el Diario Oficial el 31 de enero de 2007. Disponible en: http://www.supersalud.gob.cl/normativa/571/articles-3174_recurso_1.pdf. [Consultado el 12 de febrero de 2016].
43. Ministerio de Salud. Gobierno de Chile. Cardiopatías congénitas operables en menores de 15 años. Series guías clínicas MINSAL, 2010.

44. Weijerman ME, van Furth AM, van der Mooren MD, van Weissenbruch MM, Rammeloo L, Broers CJ, Gemke RJ. Prevalence of congenital heart defects and persistent pulmonary hypertension of the neonate with Down syndrome. Eur J Pediatr 2010; 169 (10): 1195-9.

45. Espínola-Zavaleta N, Soto ME, Romero-González A, Gómez-Puente L del C, Muñoz-Castellanos L, Gopal AS, et al. Prevalence of Congenital Heart Disease and Pulmonary Hypertension in Down's Syndrome: An Echocardiographic Study. J Cardiovasc Ultrasound 2015; 23 (2): 72-7.

46. Evans JM, Dharmar M, Meierhenry E, Marcin JP, Raff GW. Association between Down syndrome and in-hospital death among children undergoing surgery for congenital heart disease: a US population-based study. Circ Cardiovasc Qual Outcomes 2014; 7 (3): 445-52.

47. Champagne CR, Lewis M, Gilchrist DM. Should we mend their broken hearts? The history of cardiac repairs in children with Down syndrome. Pediatrics 2014; 134 (6): 1048-50. 